\begin{tabular}{llll} 
Abstract PS-299 Table 1 & & & \\
\hline Variable & GM+ve & GM-ve & p value \\
\hline Number infants & 50 & 311 & \\
GA (wk) & $26.6 \pm 3.2$ & $28.7 \pm 3.5$ & 0.0001 \\
Premature Rupture Membranes & $60 \%$ & $38 \%$ & 0.0032 \\
Prolonged Rupture Membranes $>12 \mathrm{hr}$ & $40 \%$ & $23 \%$ & 0.0047 \\
Delivery Mode C-section & $62 \%$ & $75 \%$ & 0.0492 \\
Prenatal macrolide antibiotics & $44 \%$ & $27 \%$ & 0.0143 \\
\hline
\end{tabular}

for genital mycoplasmas (GM) and complete blood counts within 24 hrs. of birth. Infants with GM+ve were compared with GMve for perinatal and neonatal variables.

Results Of the 361 infants with tracheal aspirate cultures sent for GM, 50 positive were GM+ve (See Table). Infants GM+ve had significantly higher platelet counts compared to those GMve $(285 \pm 177$ vs. $196 \pm 83 ; \mathrm{p}=0.007)$. After controlling for perinatal variables (GA $\leq 32$ wk., premature rupture of membranes, delivery mode, prenatal antibiotics and prenatal steroid) in GM+ve the adjusted odds ratio for an initial platelet count of $>250,000$ was 3.83 (95\% CI 1.5-9.8) with p value of 0.0049 . However, GM+ve infants had no significant changes in other haematological parameters such as total WBC counts, neutrophil, monocyte, lymphocyte or eosinophil counts.

Conclusions Vertical transmission of GM to the infant is manifested subtly at birth without significant changes in haematological parameters except an increase in platelet count $>250 \mathrm{~K}$.

Significance Increase in platelet counts at birth may be an important marker for the effects of GM on premature newborn infants.

\section{PS-300 CHORIAMNIONITIS AND CRP AS MARKERS FOR SHORT AND LONG TERM OUTCOMES IN PREMATURE INFANTS}

${ }^{1} E$ Ryan, ${ }^{2} D$ Eves, ${ }^{2} \mathrm{P}$ Jayadev Menon, ${ }^{2} \mathrm{~S}$ Alnafisee, ${ }^{3} \mathrm{R}$ Segurado, ${ }^{4} E$ Mooney, ${ }^{4} \mathrm{P}$ Downey, ${ }^{4}$ EJ Molloy. ${ }^{7}$ Neonatology, National Maternity Hospital, Dublin, Ireland; ${ }^{2}$ Paediatrics, Royal College of Surgeons, Dublin, Ireland; ${ }^{3}$ CSTAR, University College Dublin, Dublin, Ireland; ${ }^{4}$ Pathology, National Maternity Hospital, Dublin, Ireland

\subsection{6/archdischild-2014-307384.599}

Background Early infant CRP levels in the setting of Chorioamnionitis (CA) have not been fully defined as a predictor of cognitive, behavioural, and neuro-developmental outcomes among extremely preterm neonates.

Methods 499 preterm neonates had Placental pathology, CRP level and Vermont Oxford outcomes recorded. Death and developmental outcomes at 2 yrs were examined for 247 preterm infants $<32$ wks and $<1.5 \mathrm{~kg}$. Multivariable logistic and linear regression models were developed to assess the association between CA and outcomes controlling for gestational age.

Results 499 preterm infants were included with mean birth weight of $1074+/-273 \mathrm{~g}$ and gestational age of $28.5+/-2.7$ weeks. Infants with $\mathrm{CA}(\mathrm{N}=127)$ had lower GA and birth weight and higher rates of early onset sepsis. The Fetal inflammatory response was associated with: Apgar $<7$ at 10 mins; intubation in the delivery room; PDA; RDS; pneumothorax and oxygen on Day 28.

Infants with Day $1 \mathrm{CRP}>10 \mathrm{mg} / \mathrm{L}$ needed significantly more resuscitation in the Delivery room (DR) including adrenaline and cardiac compressions. They were significantly more at risk of RDS, ROP, grade III/IV IVH.

Conclusion There is a correlation between both initial CRP and histological CA with adverse short term outcomes.

\section{PS-301 CONDITIONS OF INTESTINAL COLONISATION IN PRETERM INFANTS DURING THE FIRST MONTH OF LIFE}

E Martín Alvarez, L Zamorano-Bonilla, MV Jiménez-Cabanillas, L Serrano-López, M PeñaCaballero, JA Hurtado-Suazo. Paediatric, Hospital Virgen de Las Nieves, Granada, Spain

\subsection{6/archdischild-2014-307384.600}

Aim To describe intestinal microbiota in preterm infants admitted to the Neonatal Unit using molecular techniques, and assess the impact of different conditions.

Methods/study design A two year period Descriptive study (2011-2012) of gut microbiota in stools from newborns borns under 35 week gestational age (WGA) at birth, admitted in a Neonatal Care Unit. Stool samples were collected: M1 (meconium), M2 (first week), M3 (first month). 5 groups of bacteria were analysed using qPCR technique: Bacteroides, Bifidobacterium, Escherichia coli, Clostridiums and Lactobacillus. Maternal, perinatal and neonate variables were registered. Statistic programs SPSSvs 20.

Results In the first month a marked increase (x120) in Bifidobacterium is observed. The increase of Lactobacillus (x2) and E. coli (x7) is lower. Bacteroides and Clostridium remain stable.

\begin{tabular}{llllll}
\multicolumn{6}{l}{ Abstract PS-301 Table 1 } \\
\hline & E coli & Clostridium & Bacteroides & Bifidobacterium & Lactobacillus \\
\hline M1 $(n=82)$ & $1,51 \times 10^{3}$ & 47,3 & 22,53 & $4,16 \times 10^{4}$ & $3,30 \times 10^{3}$ \\
M2 $(n=74)$ & $4,77 \times 10^{3}$ & 31,63 & 12,39 & $2,04 \times 10^{5}$ & $7,04 \times 10^{3}$ \\
M3 $(n=35)$ & $1,14 \times 10^{4}$ & 43,04 & 32,26 & $4,98 \times 10^{6}$ & $8,39 \times 10^{3}$ \\
\hline Value: $p 50 \mathrm{cfu} / \mathrm{g}$
\end{tabular}

Regarding WGA in $\leq 30$ vs $>30$ we observed:

- Bifidobacterial colonisation is delayed at birth $\left(2,35 \times 10^{4}\right.$ vs $\left.6,02 \times 10^{4} \mathrm{cfu} / \mathrm{g} ; \mathrm{p}=0,09\right)$ and at one week birth age $(3,98$ $\times 10^{4}$ vs $\left.1,49 \times 10^{6} \mathrm{cfu} / \mathrm{g} ; \mathrm{p}=0,007\right)$.

- Higher numbers of E. coli from the first sample $(4,37 \times$ $10^{3}$ vs $9,07 \times 10^{2} \mathrm{cfu} / \mathrm{g} ; 1,55 \times 10^{4}$ vs $2,93 \times 10^{3} \mathrm{cfu} / \mathrm{g} ; 2,94$ $\times 10^{4}$ vs $\left.9,69 \times 10^{3} \mathrm{cfu} / \mathrm{g}\right)$, although no significant statistically differences were detected.

Conclusions The number of Bifidobacterium and Lactobacillus in faecal samples is higher than the content in Bacteroides, Clostridium and E.coli. The colonisation process of studied bacteria is delayed in prematures born at lower WGA, except for E. coli.

\section{PS-302 HUMAN CATHELICIDIN ANTIMICROBIAL PEPTIDE LL37 INFLUENCES STAPHYLOCOCCUS EPIDERMIDIS' BIOFILM-ASSOCIATED GENE-EXPRESSION AND BIOFILM MASS ON A MEDICAL DEVISE SURFACE}

${ }^{1} \mathrm{E}$ Hell, ${ }^{1} \mathrm{D}$ Ujvari, ${ }^{2} \mathrm{~K}$ Hultenby, ${ }^{1} \mathrm{~K}$ Gemzell Danielsson, ${ }^{1} \mathrm{~K}$ Jansmark, ${ }^{1} \mathrm{G}$ Marchini. ${ }^{1}$ Department of Women's and Children's Health, Karolinska Institutet, Stockholm, Sweden; ${ }^{2}$ Department of Laboratory Medicine, Karolinska Institutet, Stockholm, Sweden

10.1136/archdischild-2014-307384.601 
Background and aims Device-related infections are thought to be initiated by adhesion of the bacteria to a medical device, followed by colonisation and mature biofilm formation. Preterm infants are susceptible to device-related infections caused by Staphylococcus epidermidis. Also, preterm infants have lower levels of antimicrobial peptides, including human cathelicidin antimicrobial peptide LL37, a condition that in part may explain their increased vulnerability. Our aim was to evaluate the effect of peptide LL37 on 1) the expression of biofilm-associated genes and 2) biofilm mass, by using an in vitro model.

Methods Biofilm formation of $S$. epidemidis was studied on intra vascular catheter pieces and in culture plates, in the absence or presence of LL37. Bacterial biofilm mass was investigated by scanning electron microscopy (SEM). Changes in biofilm-associated gene expression was determined by real-time polymerase chain reaction.

Results Tissue-like concentration of the peptide down-regulated most of the investigated genes after $2 \mathrm{~h}$. A diminished biofilm mass was seen on the catheter surface by SEM after $24 \mathrm{~h}$ incubation.

Conclusions Peptide LL37, as part of innate immune defense of the newborn infant is crucial for the regulation of the commensal flora, including Staphylococcus epidermidis. A diminished activity of LL37, as found in preterm infants, may contribute to increase their risk of device-related infections.

\section{PS-303 VITAMIN D INCREASES ROI PRODUCTION IN TERM AND PRETERM INFANTS: POSSIBLE MECHANISM OF ENHANCED ANTIBACTERIAL EFFECT}

${ }^{1} \mathrm{C}$ Onwuneme, ${ }^{1} \mathrm{~F}$ Martin, ${ }^{2} \mathrm{~A}$ Blanco, ${ }^{2} \mathrm{~A}$ O'Neill, ${ }^{3} \mathrm{~N}$ Murphy, ${ }^{2} \mathrm{RW}$ W Watson, ${ }^{1} \mathrm{EJ}$ Molloy. ${ }^{1}$ Neonatology, National Maternity Hospital, Dublin, Ireland; ${ }^{2}$ School of Medicine and Medical Sciences, Conway Institute University College Dublin, Dublin, Ireland; ${ }^{3}$ Paediatrics Endocrinology, Children's University Hospital Temple Street, Dublin, Ireland

\subsection{6/archdischild-2014-307384.602}

Introduction Newborn infants are at risk of vitamin D deficiency and various studies implicate vitamin D as having immunomodulatory effect. Adequate generation of reactive oxygen intermediates (ROI) by neutrophils (PMN) during sepsis is bactericidal. However, production of neutrophil oxidase activity in the presence of sepsis is impaired in neonates.

Aim To examine the in vitro effect of $1,25(\mathrm{OH})_{2} \mathrm{D}_{3}$ on whole blood PMN and monocyte ROI, TLR4, CD11b in newborn infants during sepsis in vitro.

Methods Whole blood from preterm infants $<32$ weeksgestation within $24 \mathrm{~h}$ of birth, cord blood from term infants and adult controls were analysed for phagocytic expression of Toll-Like Receptor 4 (TLR4; pathogen recognition); CD11b (chemotaxis and adhesion) and ROI production (bacterial kill) using flow cytometry. These were assessed in response to Lipopolysaccharide (LPS; Endotoxin; in vitro sepsis) and 1,25(OH) ${ }_{2} \mathrm{D}_{3}$.

Results ROI production from preterm and term neonatal neutrophils incubated with LPS alone was not significantly increased in contrast to adults. However pre-incubation with 1,25 $(\mathrm{OH})_{2} \mathrm{D}_{3}$ before adding LPS demonstrated a significant increase $(\mathrm{p}=0.001)$ in ROI production for both preterm and term infants while simultaneous LPS and 1,25(OH)2D3 had no effect. Conclusion New born infants were hypo-responsive in the presence of sepsis in vitro which recovered on pre-treatment with 1 ,
$25(\mathrm{OH})_{2} \mathrm{D}_{3}$. Pre-treatment with vitamin $\mathrm{D}$ may improve term and preterm infants' antibacterial responses.

\section{PS-304 NEONATAL ENTEROVIRUS INFECTIONS REPORTED IN FRANCE, 2012}

${ }^{1} \mathrm{~V}$ Biran, ${ }^{2}$ S Schuffenecker, ${ }^{3} \mathrm{~J}$ Aberchih, ${ }^{4} \mathrm{~L}$ Josset, ${ }^{4} \mathrm{~L}$ Bruno, ${ }^{5} \mathrm{O}$ Baud, ${ }^{5} \mathrm{~S}$ Soudée. ${ }^{1}$ Neonatal Intensive Care Unit, Robert Debré Hospital, Paris, France; ${ }^{2}$ Centre National de Référence Des Enterovirus Et Parechovirus Laboratoire de Virologie, Centre de Biologie Est, Bron, France; ${ }^{3}$ Neonatal Intensive Care Unit, Hopital Robert Debré, Paris, France; ${ }^{4}$ Centre National de Référence Des Enterovirus Et Parechovirus Laboratoire de Virologie, Centre de Biologie Est, Bron, France; ${ }^{5}$ Neonatal Intensive Care Unit, Hôpital Robert Debré, Paris, France

\subsection{6/archdischild-2014-307384.603}

Enteroviruses (EV) are among the most common viruses infecting humans. One third of EV infections concern children under 1 year. Neonatal EV infections lead to a wide range of clinical manifestations, from mild febrile illness to severe, potentially fatal sepsislike conditions with multiorgan failure.

EV detections by serotype were reported by the "National Reference Centre for Infections EV" Lyon, on a monthly basis. Demographic, clinical and biological data are also collected in neonates hospitalised in 2012 for EV infection. Two sub-groups were identified according to the beginning of symptoms before or after 8 days of life (D8). There were 120 neonatal EV infections. Before D8, children with severe infection were born more prematurely with a low birth weight. EV most commonly detected in neonates included CVB4 and E11. Risk factors of severe EV infections included liver (73\% before D8) and haematological damages (thrombocytopenia $82 \%$ and coagulopathy $64 \%$ before D8).

This study suggest a systematic serotyping of neonatal EV infections and biological monitoring of liver function to early identification of children at high risk of clinical severity and fatality.

\section{PS-305 HUMAN PARECHOVIRUS 3 AS A CAUSE OF NEONATAL INFECTION}

${ }^{1}$ A Cilla, ${ }^{2}$ J Arnaez, ${ }^{2}$ J Suarez, ${ }^{3} \mathrm{G}$ Megias, ${ }^{1} \mathrm{P}$ Alamillo, ${ }^{4} \mathrm{M}$ Cabrerizo. ${ }^{1}$ Pediatrics, Hospital Universitario de Burgos, Burgos, Spain; ${ }^{2}$ Neonatology Pediatrics, Hospital Universitario de Burgos, Burgos, Spain; ${ }^{3}$ Microbiology, Hospital Universitario de Burgos, Burgos, Spain; ${ }^{4}$ Microbiology, Centro Nacional de Microbiología Instituto de Salud Carlos III, Madrid, Spain

\subsection{6/archdischild-2014-307384.604}

Background Human parechoviruses (HPeVs) belong to Parechovirus genus and have recently been added to the Picornaviridae family. Their epidemiology, pathogenicity and virulence is only beginning to be understood. Neonates, infants and young children seem to be the most susceptible subjects. The clinical presentation is similar to that of enterovirus infections. HPeV type 3 has been reported to cause neonatal infection, presenting with central nervous system symptoms or a sepsis-like illness.

Objective As part of a prospective study on neonatal sepsis, we aimed to assess the importance of $\mathrm{HPeV}$ as a cause of infection in the neonatal period.

Materials and methods During the period October 2012 December 2014, term newborns (0-28 days) admitted to the 